\title{
Top quark decay at next-to-leading order in the standard model effective field theory
}

\author{
Radja Boughezal, ${ }^{1}$ Chien-Yi Chen, ${ }^{2}$ Frank Petriello, ${ }^{1,2}$ and Daniel Wiegand $\oplus^{1,2}$ \\ ${ }^{1}$ HEP Division, Argonne National Laboratory, Argonne, Illinois 60439, USA \\ ${ }^{2}$ Department of Physics \& Astronomy, Northwestern University, Evanston, Illinois 60208, USA
}

(Received 12 July 2019; published 30 September 2019)

\begin{abstract}
We consider top quark decay in the standard model effective field theory (SMEFT). We present a calculation of the total decay width and the $W$-boson helicity fractions at next-to-leading order (NLO) in SMEFT. Our result includes the complete set of contributing four-fermion operators in addition to QCD dipole operators and bottom-mass suppressed effects. We show that operators that first appear at NLO in the SMEFT can be bounded by the current data as well as future data from both a high-luminosity LHC and a potential $e^{+} e^{-}$collider, demonstrating the importance of going beyond leading order when studying the SMEFT. We discuss technical aspects of our calculation that we believe will be useful in future higher-order studies of the SMEFT, in particular, the treatment of $\gamma_{5}$ in loop diagrams.
\end{abstract}

DOI: 10.1103/PhysRevD.100.056023

\section{INTRODUCTION}

The standard model (SM) has so far been remarkably successful in describing all data coming from the LHC. In some channels, percent-level comparisons between theory and data are now possible. Understanding how indirect signatures of new physics are constrained by these exquisite data is becoming of central importance in extracting the most possible information from the LHC, especially given the lack of new states beyond the SM so far. This will become part of the legacy of the LHC program, similar to how the global electroweak fit became a legacy of the LEP collider. The appropriate theoretical framework for investigating these constraints is the SM effective field theory (SMEFT) containing higher-dimensional operators formed from SM fields. The leading dimension-6 operators characterizing deviations from the SM have been classified $[1,2]$ (there is a dimension-5 operator that violates lepton number which we do not consider). There has been considerable effort in performing global analyses of the available data within the framework of SMEFT [3-9].

Given the precision of the available data, it is critical to address whether the theoretical predictions entering SMEFT analysis are sufficiently precise. There are two primary considerations to address: whether higherorder corrections containing dimension- 6 operators in the

Published by the American Physical Society under the terms of the Creative Commons Attribution 4.0 International license. Further distribution of this work must maintain attribution to the author(s) and the published article's title, journal citation, and DOI. Funded by SCOAP ${ }^{3}$.
SMEFT are necessary, and whether dimension-8 operators should be considered. We consider the first of these issues in this manuscript. Within the standard model, theoretical corrections to next-to-next-to-leading order in the QCD coupling constant and next-to-leading order (NLO) in the electroweak coupling constant are known for a host of interesting processes. The situation is less advanced in the SMEFT. NLO results assuming a subset of contributing SMEFT operators are known for a host of Higgs decays [10-17] and Z-boson decays [18-20], as well as certain Higgs production processes [21,22]. Especially given the data precision, going beyond leading order (LO) is necessary to properly understand bounds on the SMEFT operators, as has been argued in the literature (see, e.g., Refs. [23,24]).

In this manuscript we study NLO corrections in the SMEFT to top quark decay. We focus on the total width and $W$-boson helicity fraction observables. There have been several analyses of constraints on the SMEFT arising from top quark data [25-30]. NLO QCD corrections to top quark decay in the SMEFT, augmented by the one-loop contribution from the top quark chromomagnetic operator, have been considered [31]. The precision of the top quark data coming from the LHC warrants these detailed investigations of top quark properties in the SMEFT. Our goals in this manuscript are summarized below.

(i) We extend the previous calculations of higher-order corrections to top quark decays in the SMEFT to also include the bottom-quark chromomagnetic dipole operator and all contributing four-fermion operators, both four-quark and semileptonic types. This is a further step toward a complete next-to-leading order 
calculation of top quark decay within the SMEFT, which we believe will eventually be warranted by the high-luminosity LHC program. It is also of phenomenological interest to determine whether third-generation four-quark operators can be constrained by this measurement. Previously suggested probes of these operators have focused on production of four external heavy-flavor states [32].

(ii) We emphasize the role of chiral Ward identities as an important calculational check, in particular, in the treatment of $\gamma_{5}$ in loops containing four-fermion operators. We consider several different schemes for the treatment of $\gamma_{5}$ in dimensional regularization and demonstrate how imposing chiral Ward identities renders them consistent. We believe that this discussion will be useful in the future as higher-order effects in the SMEFT are further studied.

(iii) We study the effect of bottom-quark mass-suppressed contributions in the SMEFT. Interestingly, such effects go like $m_{b} / m_{t}$ in the SMEFT at LO due to the chiral structure of the contributing dimension-6 operators, unlike in the SM where they go as $\left(m_{b} / m_{t}\right)^{2}$. This leads to significant constraints on these operators from current data.

(iv) We consider the constraints on all operators using the currently available data on the total width and $W$-helicity fractions. We also derive simple projections for a high-luminosity LHC and a possible future $e^{+} e^{-}$machine. Our primary goal is to determine how well loop-induced operators can be probed given both current and future experimental measurements. As we consider only a subset of the available data rather than perform a global fit as in $[27,29,30]$, our numerical results should only be considered representative of the potential bounds.

Our paper is organized as follows. In Sec. II we provide a brief overview of the SMEFT operators relevant for our calculation of top quark decay. We present the leadingorder calculation of our observables in Sec. III, establishing our calculational framework. We present our NLO calculation in Sec. IV. We discuss in detail the technical aspects of the calculation particular to the SMEFT such as the treatment of $\gamma_{5}$ and the ultraviolet (UV) renormalization. Our numerical results are shown in Sec. V. Finally, we conclude in Sec. VI.

\section{OVERVIEW OF TOP QUARK DECAY IN SMEFT}

We begin by discussing the features of the dimension- 6 SMEFT relevant to our calculation of top quark decay, $t \rightarrow W b$. At leading order this process proceeds through the single Feynman diagram shown in Fig. 1. To determine the SMEFT contributions to this process we use the Warsaw basis [1]. Following the notation of Ref. [33] we find the following operators contributing at leading order:

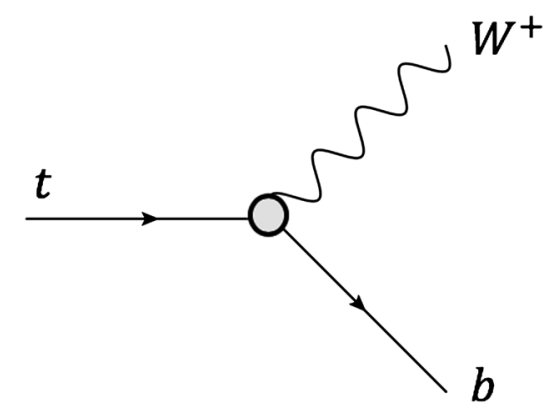

FIG. 1. Leading-order Feynman diagram for the process $t \rightarrow W b$. Through the shaded vertex the Wilson coefficients $C_{t W}, C_{b W}$, and $C_{t b \phi}$ enter the amplitude.

$$
\begin{aligned}
\mathcal{O}_{p w}=\bar{q}_{p} \sigma^{\mu \nu} u_{r} \tau^{I} \tilde{\phi} W_{\mu \nu}^{I}, \\
\mathcal{O}_{\substack{d W \\
p r}}=\bar{q}_{p} \sigma^{\mu \nu} d_{r} \tau^{I} \phi W_{\mu \nu}^{I}, \\
\mathcal{O}_{p u d}=i\left(\tilde{\phi}^{\dagger} D_{\mu} \phi\right)\left(\bar{u}_{p} \gamma^{\mu} d_{r}\right) .
\end{aligned}
$$

Here, $q_{p}$ denotes the left-handed quark doublet with $p$ being the generation index, $u_{r}$ and $d_{r}$ are respectively the up and down right-handed singlet quarks with generation index $r, \phi$ is the Higgs doublet, and $W_{\mu \nu}^{I}$ is the field-strength tensor for the $S U(2)_{L}$ gauge bosons with $I$ denoting the isospin index. $\sigma_{\mu \nu}$ is written in terms of the commutator of $\gamma$ matrices as $\sigma_{\mu \nu}=i\left[\gamma_{\mu}, \gamma_{\nu}\right] / 2$. These operators are written in the flavor eigenstate basis. Rotating to the mass basis introduces mixing matrices into the Wilson coefficient matrices multiplying these operators. In our analysis we restrict ourselves to third-generation couplings, and study the operators

$$
\mathcal{O}_{t W}=\mathcal{O}_{u W}, \quad \mathcal{O}_{b W}=\mathcal{O}_{d W}, \quad \mathcal{O}_{\phi t b}=\mathcal{O}_{\text {कud }} .
$$

We label the Wilson coefficients multiplying these operators as $C_{t W}, C_{b W}$, and $C_{\phi t b}$, respectively, and assume for simplicity that they are real. We factor out the energy scale $1 / \Lambda^{2}$ associated with these operators being dimension 6 so that the Wilson coefficients $C_{i}$ are dimensionless.

In order to illustrate the sensitivity of top quark decay properties to NLO effects in SMEFT we consider a subset of the operators that contribute at NLO. As we show later explicitly, and as can be seen using the renormalization group equations of SMEFT, a consistent NLO calculation using the operators above requires the following QCD dipole operators:

$$
\begin{aligned}
& \mathcal{O}_{p r}=\bar{q}_{p} \sigma^{\mu \nu} T^{A} u_{r} \tilde{\phi} G_{\mu \nu}^{A}, \\
& \mathcal{O}_{\substack{d g \\
p r}}=\bar{q}_{p} \sigma^{\mu \nu} T^{A} d_{r} \phi G_{\mu \nu}^{A} .
\end{aligned}
$$

Here, $G_{\mu \nu}^{A}$ is the gluon field-strength tensor and $T^{A}$ are the color matrices in the fundamental representation. We again 
restrict our analysis to third-generation couplings and study the operators

$$
\mathcal{O}_{t g}=\mathcal{O}_{u g}, \quad \mathcal{O}_{b g}=\mathcal{O}_{\substack{d g \\ 33}}
$$

We also consider the following four-fermion operators which potentially contribute to top decay as well. In the case of four-quark operators we have

$$
\begin{aligned}
& \mathcal{O}_{q q}^{(1)}=\left(\bar{q}_{p} \gamma^{\mu} q_{r}\right)\left(\bar{q}_{s} \gamma_{\mu} q_{t}\right) \quad \mathcal{O}_{q q}^{(3)}=\left(\bar{q}_{p} \gamma^{\mu} \tau^{a} q_{r}\right)\left(\bar{q}_{s} \gamma_{\mu} \tau^{a} q_{t}\right) \\
& \mathcal{O}_{u d}^{(1)}=\left(\bar{u}_{p} \gamma^{\mu} u_{r}\right)\left(\bar{d}_{s} \gamma_{\mu} d_{t}\right) \quad \mathcal{O}_{u d}^{(8)}=\left(\bar{u}_{p} \gamma^{\mu} T^{A} u_{r}\right)\left(\bar{d}_{s} \gamma_{\mu} T^{A} d_{t}\right) \\
& \mathcal{O}_{q u}^{(1)}=\left(\bar{q}_{p} \gamma^{\mu} q_{r}\right)\left(\bar{u}_{s} \gamma_{\mu} u_{t}\right) \quad \mathcal{O}_{q u}^{(8)}=\left(\bar{q}_{p} \gamma^{\mu} T^{A} q_{r}\right)\left(\bar{u}_{s} \gamma_{\mu} T^{A} u_{t}\right) \\
& \mathcal{O}_{q d}^{(1)}=\left(\bar{q}_{p} \gamma^{\mu} q_{r}\right)\left(\bar{d}_{s} \gamma_{\mu} d_{t}\right) \quad \mathcal{O}_{q d}^{(8)}=\left(\bar{q}_{p} \gamma^{\mu} T^{A} q_{r}\right)\left(\bar{d}_{s} \gamma_{\mu} T^{A} d_{t}\right) \\
& \mathcal{O}_{q u q d}^{(1)}=\left(\bar{q}_{p}^{j} u_{r}\right) \epsilon_{j k}\left(\bar{q}_{s}^{k} d_{t}\right) \quad \mathcal{O}_{q u q d}^{(8)}=\left(\bar{q}_{p}^{j} T^{A} u_{r}\right) \epsilon_{j k}\left(\bar{q}_{s}^{k} T^{A} d_{t}\right) \text {, }
\end{aligned}
$$

where $\tau^{a}$ are the Pauli matrices and summation over the $S U(2)$ index $a$ is implied. For simplicity we consider only flavor-diagonal operators in our study; constraints on flavor-violating operators are generally better obtained from other observables than those considered here. We also include the following semileptonic four-fermion operators in our analysis:

$$
\begin{aligned}
\mathcal{O}_{l q}^{(3)} & =\left(\bar{l}_{p} \gamma^{\mu} \tau^{a} l_{r}\right)\left(\bar{q}_{s} \gamma_{\mu} \tau^{a} q_{t}\right) \\
\mathcal{O}_{\text {lequ }}^{(3)} & =\left(\bar{l}_{p}^{j} \sigma^{\mu \nu} e_{r}\right) \epsilon_{j k}\left(\bar{q}_{s}^{k} \sigma_{\mu \nu} u_{t}\right) .
\end{aligned}
$$

It is necessary for the one-loop renormalization of SMEFT to include the following operator as well:

$$
\mathcal{O}_{\phi q}^{(3)}=i\left(\Phi^{\dagger} \stackrel{\leftrightarrow}{D}_{\mu}^{a} \Phi\right)\left(\bar{q}_{p} \tau^{a} \gamma^{\mu} q_{r}\right),
$$

which leads to a redefinition of the CKM matrix:

$$
K_{\mathrm{CKM}} \rightarrow K_{\mathrm{CKM}}\left(1+\frac{v^{2}}{\Lambda^{2}} C_{\phi q}^{(3)}\right) .
$$

At leading order this generates a term proportional to the SM Born-level matrix element. As pointed out in [34-36], the poles associated with the corrections from four-fermion operators to the $t b W$-vertex are removed by renormalizing $C_{\phi q}^{(3)}$. This leads us to the following Lagrangian describing top decay,

$$
\mathcal{L}=\mathcal{L}_{\mathrm{SM}}+\frac{1}{\Lambda^{2}} \sum_{i} C_{i} \mathcal{O}_{i}
$$

with $i$ running over all operators previously discussed. All Feynman rules arising from these operators can be found in Ref. [33].
We note that electroweak operators that first appear at NLO are neglected in our analysis. Such contributions are far better bounded through other data sets such as precision $Z$-pole observables. To see this, we consider a representative example of such an operator, $\mathcal{O}_{\phi B}$. Reference [19] found the following bound on the Wilson coefficient associated with this operator from precision $Z$-pole data: $\left|C_{\phi B}\right|<0.0018$. We see in our numerical results section that the bounds we obtain on Wilson coefficients that first appear at NLO are far weaker. For this reason we have restricted our analysis to NLO operators specific to the top quark sector of the SMEFT.

\section{LEADING-ORDER CALCULATION}

We discuss here some basic features of our calculation and present results for the LO top decay width and helicity fractions in the SMEFT. The only diagram mediating the decay at tree level is shown in Fig. 1. It is straightforward to derive the amplitude for the decay $t\left(p_{t}\right) \rightarrow b\left(p_{b}\right) W\left(p_{W}\right)$ using the operators of Sec. II. We consider four observables: the total top decay width $\Gamma_{\text {tot }}$, and the decay fractions into longitudinal, positive, and negative $W$-boson helicities. To obtain these quantities from the decay amplitude it is convenient to replace the $W$-boson polarization vectors in the squared amplitude according to

$$
\sum \epsilon^{\mu}\left(p_{W}\right) \epsilon^{* \nu}\left(p_{W}\right)=P^{\mu \nu}
$$

We use the following projection operators [37]:

$$
\begin{aligned}
& P_{\mathrm{tot}}^{\mu \nu}=-g^{\mu \nu}+\frac{p_{W}^{\mu} p_{W}^{\nu}}{M_{W}^{2}}, \\
& P_{\mathrm{L}}^{\mu \nu}=\frac{\left[M_{W}^{2} p_{t}^{\mu}-p_{t} \cdot p_{W} p_{W}^{\mu}\right]\left[M_{W}^{2} p_{t}^{\nu}-p_{t} \cdot p_{W} p_{W}^{\nu}\right]}{M_{W}^{2} m_{t}^{2}\left|\vec{p}_{W}\right|^{2}}, \\
& P_{\mathrm{F}}^{\mu \nu}=-\frac{i}{m_{t}\left|\vec{p}_{W}\right|} \epsilon^{\mu \nu \sigma \rho} p_{t \sigma} p_{W \rho}, \\
& P_{ \pm}^{\mu \nu}=\frac{1}{2}\left\{P_{\mathrm{tot}}^{\mu \nu}-P_{\mathrm{L}}^{\mu \nu} \pm P_{\mathrm{F}}^{\mu \nu}\right\} .
\end{aligned}
$$

$\vec{p}_{W}$ denotes the three-momentum of the $W$ boson. We use these projectors in both the LO and NLO calculations.

In our calculation we only include terms linear in the EFT couplings, as terms proportional to EFT couplings squared are of the same order as neglected dimension-8 operators. We include finite bottom-mass effects at leading order. We present below the top decay width and helicity fractions in the SMEFT. For simplicity of presentation we have expanded them to linear order in $m_{b} / m_{t}$ (in our numerical analysis we keep the LO results to all orders in this ratio): 


$$
\begin{aligned}
\Gamma_{\text {tot }}= & \frac{\bar{g}\left(x_{W}^{2}-1\right) m_{t}}{64 \pi x_{W}}\left[\left(x_{W}^{2}-1\right)\left(\bar{g}\left(1+2 x_{W}^{2}\right)+12 \sqrt{2} C_{t W} m_{t}^{2} x_{v} x_{W}^{2}\right)\right. \\
& \left.+6 m_{t}^{2} x_{b} x_{v} x_{W}^{2}\left(C_{\phi t b} \bar{g} x_{v}+2 \sqrt{2} C_{b W}\left(1+x_{W}^{2}\right)\right)\right]+\mathcal{O}\left(x_{b}^{2}\right), \\
F_{\mathrm{L}}^{\mathrm{LO}}= & \frac{\Gamma_{\mathrm{L}}}{\Gamma_{\text {tot }}}=\frac{4 \sqrt{2} m_{t}^{2} x_{v}\left(x_{W}^{2}-1\right) x_{W}^{2}\left(2 C_{t W}+\bar{g}\left(2 x_{W}^{2}+1\right)\right)}{\bar{g}\left(2 x_{W}^{2}+1\right)^{2}} \\
& +\frac{4 \sqrt{2} m_{t}^{2} x_{v} x_{b} x_{W}^{2}\left(2 C_{b W}\left(x_{W}^{2}+1\right)+\frac{\bar{g} x_{v}}{\sqrt{2}} C_{\phi t b}\left(x_{W}^{2}+1\right)\right)}{\bar{g}\left(2 x_{W}^{2}+1\right)^{2}}+\mathcal{O}\left(x_{b}^{2}\right), \\
F_{-}^{\mathrm{LO}}= & \frac{\Gamma_{-}}{\Gamma_{\text {tot }}}=\frac{2 x_{W}^{2}\left(\bar{g}+2 \bar{g} x_{W}^{2}+4 \sqrt{2} C_{t W} m_{t}^{2} x_{v}\left(1-x_{W}^{2}\right)\right)}{\bar{g}\left(1+2 x_{W}^{2}\right)^{2}} \\
& +\frac{2 m_{t}^{2} x_{b} x_{v} x_{W}^{2}\left(4 \sqrt{2} C_{b W} x_{W}^{2}\left(2+x_{W}^{2}\right)-C_{\phi t b} \bar{g} x_{v}\left(1-4 x_{W}^{2}\right)\right)}{\bar{g}\left(1+3 x_{W}^{2}-4 x_{W}^{6}\right)}+\mathcal{O}\left(x_{b}^{2}\right) .
\end{aligned}
$$

We have abbreviated $x_{i}=m_{i} / m_{t}$ and $x_{v}=v / m_{t}$, where $v$ is the Higgs vacuum expectation value. $\Gamma_{i}$ denotes the partial decay widths for the different W polarizations. $\bar{g}$ is the scaled electroweak coupling required to canonically normalize the gauge fields in SMEFT [33]. We note that the positive helicity fraction can be obtained using the relation $F_{+}=1-F_{-}-F_{L}$. This relation can be easily seen to hold using the projectors of Eq. (12). These quantities reduce to the known SM results [37] when all SMEFT Wilson coefficients are set to 0 .

One interesting feature of these results is their dependence on the bottom-quark mass fraction $x_{b}$. In the SM the dependence on the bottom quark mass at Born level begins at $\mathcal{O}\left(x_{b}^{2}\right)$, while in SMEFT it begins at $\mathcal{O}\left(x_{b}\right)$. This is because the EFT operators $\mathcal{O}_{b W}$ and $\mathcal{O}_{\phi t b}$ have a $V+A$ helicity structure instead of the $V-A$ structure of the SM. We see the effect of this parametric difference in our numerical results.

\section{NEXT-TO-LEADING-ORDER CALCULATION}

We discuss in this section our calculation of the NLO corrections to top quark decay properties in the SMEFT. Higher-order QCD-like corrections involving gluon exchange are mediated by both SM QCD and the operators listed in Sec. II giving rise to the diagrams in Fig. 2. Contributions from the four-fermion operators listed in Eqs. (6) and (7) give rise to the Feynman diagrams shown in Fig. 3. Corrections arising from the electroweak sector in the SM are known to be subdominant [38] and are neglected in this study. In the NLO corrections we neglect the bottom mass dependence. Most aspects of this calculation are completely standard. For the one-loop virtual corrections we use integration-by-parts identities [39] to reduce all integrals to master integrals. For this calculation only the one-loop tadpole and one-loop bubble integral with a single massive internal line are needed, and are trivial to obtain. Real radiation corrections required for the QCD corrections are obtained from the process $t \rightarrow b W g$. These are straightforward to integrate over the final-state phase space to obtain the total decay width and helicity fractions. The four-fermion corrections are infrared finite and only require UV renormalization. We regulate all ultraviolet and infrared divergences appearing in intermediate stages using conventional dimensional regularization. The final analytic results are presented in the Appendix. In the following subsections we focus on technical aspects of the calculation specific to the SMEFT.

\section{A. Treatment of $\gamma_{5}$ in SMEFT}

The appearance of $\gamma_{5}$ in the Feynman rules for this process indicates that a prescription for handling this

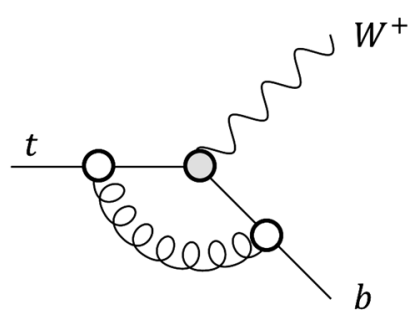

(a)

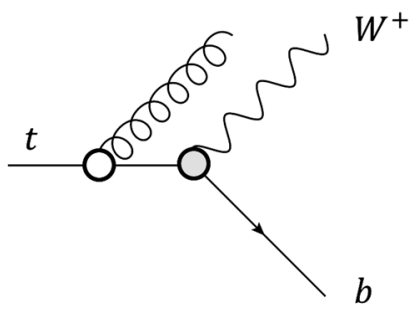

(b)

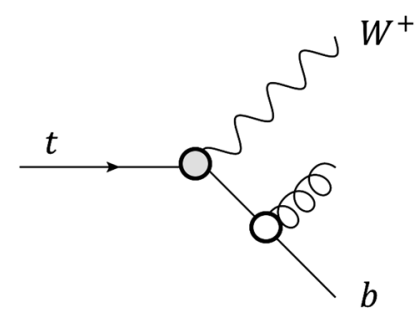

(c)

FIG. 2. Feynman diagrams for the SMEFT QCD corrections to $t \rightarrow W b$. Through the white vertices the Wilson coefficients $C_{t g}$ and $C_{b g}$ enter the amplitude. The expansion in $\frac{1}{\Lambda}$ happens at the squared matrix-element level. 


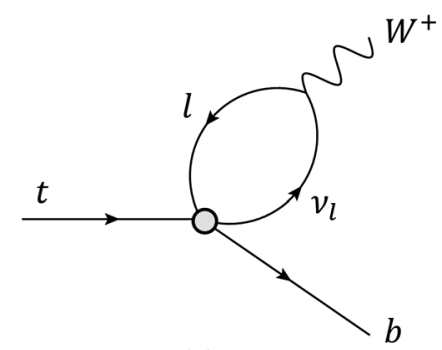

(a)

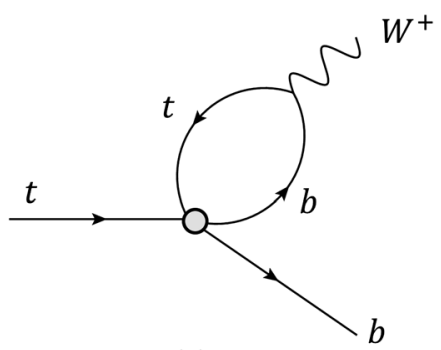

(b)

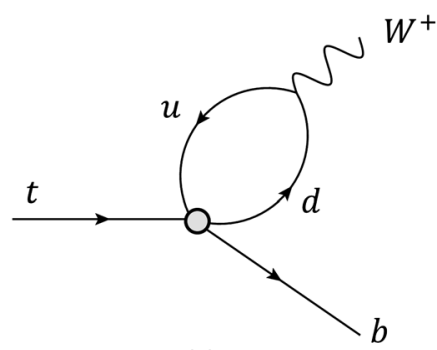

(c)

FIG. 3. Feynman diagrams for the four-fermion SMEFT corrections to $t \rightarrow W b$. Through the shaded vertices the Wilson coefficients enter the amplitude. The vertex involving the $W$ boson receives in principle contributions from SMEFT operators as well, but since the four-fermion vertex has a vanishing SM limit those would lead to terms of order $\frac{1}{\Lambda^{4}}$ and are therefore neglected.

quantity when the space-time dimension $d=4-2 \epsilon$ is needed. Different ways of treating $\gamma_{5}$ have been extensively discussed in the literature; for a review see Ref. [40]. In our calculation $\gamma_{5}$ appears in three places: through the axialvector current insertion $\gamma_{\mu} \gamma_{5}$, through the axial-tensor current $\sigma_{\mu \nu} \gamma_{5}$, and through the Dirac structure of each four-fermion operator.

\section{An overview of chiral Ward identities}

In the massless limit the SM portion of the Lagrangian of Eq. (10) has the following chiral U(2) symmetry:

$$
\begin{aligned}
& q_{L} \rightarrow e^{\frac{i}{2} \alpha_{i} \tau_{i}} q_{L} \\
& q_{R} \rightarrow e^{-\frac{i}{2} \alpha_{i} \tau_{i}} q_{R},
\end{aligned}
$$

where $q_{L, R}=\left(t_{L, R}, b_{L, R}\right)$ denote the left-handed and righthanded third-generation quark doublet. $\tau_{i}$ are the generators of this symmetry; $\tau_{0}$ is the identity matrix while the $\tau_{i}$ are the Pauli matrices rescaled by a factor of $\frac{1}{2}$. In the SM this symmetry is broken by the quark masses (in this study we consider only a nonzero top quark mass). This leads to well-known relations between the divergence of the axialvector current and the pseudoscalar current as summarized in [41], and consequently between correlation functions in the theory. Any prescription for $\gamma_{5}$ in dimensional regularization must satisfy these relations.

The situation in the SMEFT is slightly more complicated, as not all of the operators of Sec. II satisfy the symmetry of Eq. (14). Operators such as $\mathcal{O}_{q q}^{(1)}$ which are formed from the doublets $q_{L}$ satisfy the symmetry. Those such as $\mathcal{O}_{q u}^{(1)}$ which feature explicit top or bottom quarks, or those such as $\mathcal{O}_{t g}$ which couple left-handed states to righthanded ones, do not. The standard chiral Ward identities must be modified in the presence of such operators.

In our calculation we make use of several different schemes for $\gamma_{5}$, and check both their consistency with each other and that they satisfy the appropriate chiral Ward identities. This gives us confidence that our treatment of $\gamma_{5}$ is correct. We summarize below what schemes are used in our calculation. More details appear in the following subsections.

(i) For the QCD-like operators a convenient scheme due to Larin [42] is available in the literature. We use this approach together with several internal checks to ensure correctness of our results.

(ii) For the four-fermion operators we use both a naive anticommuting scheme, which is expected to lead to consistent results in this calculation [41], and the 't Hooft-Veltman-Breitenlohner-Maison (HVBM) scheme [43]. We demonstrate that both satisfy the chiral Ward identities after appropriate renormalization and lead to identical results.

We note that there are other consistent schemes that lead to equivalent results, such as Refs. [44,45] which abandon the cyclicity of the trace. We do not consider these alternatives here.

\section{QCD-like operators: the Larin scheme}

The SM and the QCD-like operators of Eq. (3) contain both the axial-vector current and the axial-tensor current. The axial-vector current appears in the SM, and its treatment in dimensional regularization has been studied extensively. A convenient way to treat the axial vector current is due to Larin [42], and involves the following replacement:

$$
\gamma_{\mu} \gamma_{5} \rightarrow \frac{i}{6} \epsilon_{\mu \nu \rho \sigma} \gamma^{\nu} \gamma^{\rho} \gamma^{\sigma}
$$

The indices appearing in the Levi-Civita symbol are treated as $d$-dimensional indices. This replacement violates the chiral Ward identities outlined above, leading to the need for an additional finite renormalization factor:

$$
Z_{5}^{n s}=1-\frac{\alpha_{s} C_{F}}{\pi}+\mathcal{O}\left(\alpha_{s}^{2}\right) .
$$

It is straightforward to check that $\gamma_{5}$ defined by Eq. (15) no longer anticommutes with $\gamma_{\mu}$ when $\mu$ extends beyond four dimensions. In our calculation we encounter Dirac traces with either two factors of $\gamma_{5}$ or a single $\gamma_{5}$. In the first case we can replace the axial-vector current according to 
Eq. (15) immediately at the level of the Feynman rules, or assume an anticommuting $\gamma_{5}$ in order to remove them completely from the trace, removing the need for $Z_{5}^{n s}$. We find that both treatments lead to the same final answer, consistent with the discussion in Ref. [40]. This serves as a check of our procedure. We also reproduce exactly the known SM QCD results for the total width and helicity fractions.

The axial-tensor current does not appear in the standard model Feynman rules. We note that in $d=4$, the Chisholm identity can be used to rewrite the axial-tensor current according to

$$
\sigma_{\mu \nu} \gamma_{5}=-\frac{i}{2} \epsilon_{\mu \nu \rho \sigma} \sigma^{\rho \sigma}
$$

By avoiding the ${ }^{1}$ introduction of a nonanticommuting $\gamma_{5}$ all Ward identities are preserved, indicating that no additional finite renormalization is needed for the axial-tensor current, unlike for the axial-vector current. This observation has also been made in previous studies in heavy-quark effective theory [46]. To check this result we have also used the 't Hooft-Veltman replacement

$$
\gamma_{5} \rightarrow \frac{i}{24} \epsilon_{\mu \nu \rho \sigma} \gamma^{\mu} \gamma^{\nu} \gamma^{\rho} \gamma^{\sigma}
$$

and have found the same final result as obtained with the Chisholm replacement. We note that the Chisholm replacement is computationally more efficient, as it leads to fewer $\gamma$-matrices within Dirac traces.

To have an independent check of the validity of the Larin scheme in the presence of SMEFT operators we verify the corresponding chiral Ward identities through explicit calculation. We consider $\mathcal{O}_{t g}$ as an example. This operator violates the chiral $\mathrm{U}(2)$ symmetry since it contains a current coupling a left-handed and right-handed state. A variation of the Lagrangian under the symmetry transformation considered leads to the following relation between correlation functions:

$$
\begin{aligned}
& \left\langle\partial_{\mu}\left(\bar{t}(x) \gamma^{\mu} \gamma_{5} t(x)\right) t\left(x_{1}\right) \bar{t}\left(x_{2}\right)\right\rangle \\
& =2 i m_{t}\left\langle\bar{t}(x) \gamma_{5} t(x) t\left(x_{1}\right) \bar{t}\left(x_{2}\right)\right\rangle \\
& \quad-i \gamma_{5}\left\langle t(x) \bar{t}\left(x_{2}\right)\right\rangle \delta^{d}\left(x-x_{1}\right)-i\left\langle t\left(x_{1}\right) \bar{t}(x)\right\rangle \gamma_{5} \delta^{d}\left(x-x_{2}\right) \\
& \quad-\sqrt{2} i \frac{v}{\Lambda^{2}} C_{t g}\left\langle\left(\bar{t}(x) \sigma^{\mu \nu} \gamma_{5} T^{A} t(x) G_{\mu \nu}^{A}\right) t\left(x_{1}\right) \bar{t}\left(x_{2}\right)\right\rangle .
\end{aligned}
$$

The derivation implicitly assumes that the functional measure transforms trivially under the chiral rotation; i.e., the symmetry is nonanomalous. This assumption is supported by discussions in [47], which indicate that any anomalous terms are proportional to the square of the

\footnotetext{
${ }^{1}$ The conventions here are such that $\gamma_{5}=i \gamma^{0} \gamma^{1} \gamma^{2} \gamma^{3}$ and $\epsilon^{0123}=+1$.
}

Wilson coefficients. The left- and right-handed side of the identity agree with each other in the Larin scheme described above, giving us confidence in our results for the decay widths calculated in SMEFT.

Since our top decay calculation does not involve traces over triple axial vector current insertions that appear in triangle fermion loops, we can extend the indices of LeviCivita symbol safely from 4 to $d$ dimensions. We have verified through explicit calculation that all differences in this treatment of the Levi-Civita symbol in combination with both the Chisholm replacement as well as the 't Hooft-Veltman replacement appear only at $\mathcal{O}(\epsilon)$ for our observables.

\section{Four-Fermion operators: naive anticommuting and $\mathrm{HVBM}$ schemes}

In the case of diagrams involving four-fermion interactions we follow a two-pronged approach to obtain consistent results. First we employ the FEYNCALC [48] internal scheme in which an anticommuting $\gamma_{5}$ is assumed in combination with

$$
\operatorname{Tr}\left[\gamma_{5} \gamma^{\mu} \gamma^{\nu} \gamma^{\rho} \gamma^{\sigma}\right]=4 i \epsilon^{\mu \nu \rho \sigma}
$$

where the Levi-Civita symbol is treated as a strictly fourdimensional object. Combining an anticommuting $\gamma_{5}$ with a nonvanishing trace as done here is strictly speaking inconsistent but is known to lead to correct results in the case of one-loop corrections [49]. Since there exists no formal proof for this statement we verify our results again by confirming that the scheme preserves the Ward identities between correlation functions associated with Eq. (14), which in the presence of the four-fermion operators reads

$$
\begin{aligned}
\left\langle\partial_{\mu}\left(\bar{t}(x) \gamma^{\mu} \gamma_{5} t(x)\right) t\left(x_{1}\right) \bar{t}\left(x_{2}\right)\right\rangle= & 2 i m_{t}\left\langle\bar{t}(x) \gamma_{5} t(x) t\left(x_{1}\right) \bar{t}\left(x_{2}\right)\right\rangle \\
& -i \gamma_{5}\left\langle t(x) \bar{t}\left(x_{2}\right)\right\rangle \delta^{d}\left(x-x_{1}\right) \\
& -i\left\langle t\left(x_{1}\right) \bar{t}(x)\right\rangle \gamma_{5} \delta^{d}\left(x-x_{2}\right),
\end{aligned}
$$

and analogously for the bottom-quark current. We confirm through explicit calculation that the naive anticommuting scheme, as implemented in FEYNCALC, preserves the Ward identity in the presence of four-fermion operators that conserve the chiral $U(2)$ symmetry. As mentioned previously some operators (e.g., $C_{q u}^{(1)}$ ) explicitly violate the chiral transformation, and consequently satisfy a more complicated identity. As a second check we employ the selfconsistent HVBM scheme [43], as it is implemented in TRACER [50]. This involves splitting all $d$-dimensional objects into sums of their four-dimensional parts (denoted by a bar) and $d$-4-dimensional (denoted by a hat) parts,

$$
g^{\mu \nu}=\bar{g}^{\mu \nu}+\hat{g}^{\mu \nu} \quad q^{\mu}=\bar{q}^{\mu}+\hat{q}^{\mu},
$$


where external momenta and the Levi-Civita symbol are treated as purely four dimensional. We note that each of the bared and hatted objects acts as a projector for the 4 and $d$-4-dimensional subspaces, respectively. The results obtained in this approach violate the chiral Ward identities which need to be restored through the introduction of finite corrections stemming from evanescent operators, as described in $[41,51,52]$. After the inclusion of these finite corrections the results obtained with this approach must agree through $\mathcal{O}\left(\epsilon^{0}\right)$ with those obtained using FEYNCALC. We have checked for several operators under consideration that this is indeed the case.

\section{B. Ultraviolet renormalization}

The UV renormalization of the external states is performed in the on-shell scheme, similar to the renormalization usually performed in the standard model. The only nonvanishing terms stem from the QCD corrections to the external top quark line. The quark self-energy corrections from the four-fermion operators are independent of their respective momenta and therefore do not change the wave function renormalization. Electric charge, weak mixing angle and $W$-wave function do not receive any contributions, since they exclusively depend on the gauge-boson self energies.

To calculate the gluonic contribution to the wave function renormalization we note that the quark self energy can be decomposed in SMEFT in the same way as in the SM,

$$
\Sigma_{q}\left(p^{2}\right)=\not p P_{L} \Sigma_{q}^{L}\left(p^{2}\right)+\not p P_{R} \Sigma_{q}^{R}\left(p^{2}\right)+m_{t} \Sigma_{q}^{S}\left(p^{2}\right),
$$

with the chirality projection operators $P_{R / L}=\frac{1}{2}\left(1 \pm \gamma_{5}\right)$. We therefore can calculate the left- and right-handed quark field renormalizations $\delta Z_{q}^{L / R}$ from the quark self energy according to

$$
\begin{aligned}
\delta Z_{q}^{R / L}= & -\Sigma_{q}^{R / L}\left(m_{t}^{2}\right) \\
& -\left.m_{q}^{2} \frac{\partial}{\partial p^{2}} \operatorname{Re}\left\{\Sigma_{q}^{L}\left(p^{2}\right)+\Sigma_{q}^{R}\left(p^{2}\right)+2 \Sigma_{q}^{S}\left(p^{2}\right)\right\}\right|_{p^{2}=m_{q}^{2}} .
\end{aligned}
$$

Calculating this expression in the SMEFT for the top quark yields

$\delta Z_{t}^{R / L}=\frac{C_{F} g_{s}}{32 \pi^{2}} \frac{d-1}{d-3} \frac{A_{0}\left(m_{t}^{2}\right)}{m_{t}^{2}}\left[g_{s}(d-2)-2 \sqrt{2} x_{v} m_{t}^{2} C_{t g}\right]$.

Our conventions are such that the tadpole master integral is

$$
A_{0}\left(m^{2}\right)=m^{2}\left[\frac{1}{\epsilon}+1-\log \left(\frac{m^{2}}{\mu^{2}}\right)\right]
$$

with renormalization scale $\mu$, which is in the end set to the top mass $m_{t}$ in our numerical studies. This also explicitly confirms that left- and right-handed top quarks still receive the same contributions in SMEFT QCD. Furthermore, the corresponding field renormalizations of the bottom quark vanish identically in the limit $m_{b}=0$.

It is necessary to introduce additional counterterms by renormalizing the SMEFT Wilson coefficients themselves. This is customarily done in $\overline{\mathrm{MS}}$ [34-36] and can be achieved in the QCD sector through the replacement

$$
\begin{aligned}
C_{t W} & \rightarrow C_{t W}+\frac{C_{F} g_{s}^{2}}{16 \pi^{2} \epsilon} C_{t W}-\frac{C_{F} \bar{g} g_{s}}{16 \pi^{2} \epsilon} C_{t g} \\
C_{b W} & \rightarrow C_{b W}+\frac{C_{F} g_{s}^{2}}{16 \pi^{2} \epsilon} C_{b W}-\frac{C_{F} \bar{g} g_{s}}{16 \pi^{2} \epsilon} C_{b g},
\end{aligned}
$$

introducing further operator mixing. In the case of the fourfermion operators we renormalize the operator $C_{\phi q}^{(3)}$ by shifting the CKM matrix as mentioned before. We choose

$$
\delta C_{\phi q}^{(3)}=\frac{\bar{g}^{2} C_{l q}^{(3)}}{48 \pi^{2} \epsilon},
$$

for a lepton pair $l, \nu_{l}$ in the loop, as well as

$$
\delta C_{\phi q}^{(3)}=\frac{\bar{g}^{2}-3 y_{t}^{2}}{48 \pi^{2} \epsilon}\left(C_{q q}^{(1)}+\left(2 N_{C}-1\right) C_{q q}^{(3)}\right),
$$

for the $t, b$ loop, where the nonvanishing top mass in the loop gives rise to the top Yukawa $y_{t}=\frac{\sqrt{2} m_{t}}{v}$. Correspondingly we find

$$
\delta C_{\phi q}^{(3)}=\frac{\bar{g}^{2}}{48 \pi^{2} \epsilon}\left(C_{q q, \mathrm{light}}^{(1)}+\left(2 N_{C}-1\right) C_{q q, \mathrm{light}}^{(3)}\right),
$$

for light quarks in the loop. We report the counterterms here for completeness but omit the light quark loop from our analysis, since the associated Dirac structure could only be achieved by integrating out a heavy neutral vector boson that changes quark flavor from a UV completion. The counterterms found here are in agreement with the ones reported in $[35,36]$. We note that for consistency $\alpha_{s}$ is run from the $Z$-scale up to the top mass scale utilizing the two loop SM-running found in the literature [53].

\section{NUMERICAL RESULTS}

We present our numerical results in this section. We assume $\Lambda=500 \mathrm{GeV}$ throughout this section, which makes the Wilson coefficients under discussion dimensionless. The input parameters are summarized in Table I. The measured values of the top decay width and helicity fractions we use to constrain the operators are taken from the PDG [54], 
TABLE I. Input parameters for the calculation, taken from [54]. The value of the $S U(2)$ coupling $\bar{g}$ is calculated from the Fermi constant $G_{F}$ and the fine structure constant $\alpha_{\mathrm{em}}$.

\begin{tabular}{lccc}
\hline \hline$M_{Z}$ & $91.1876 \mathrm{GeV}$ & $M_{W}$ & $80.379 \mathrm{GeV}$ \\
$v$ & $246 \mathrm{GeV}$ & $m_{t}$ & $173.0 \mathrm{GeV}$ \\
$m_{b}$ & $4.78 \mathrm{GeV}$ & $G_{F}$ & $1.1664 \times 10^{-5} \mathrm{GeV}^{-2}$ \\
$\alpha_{\mathrm{em}}^{-1}$ & 137.036 & $\alpha_{s}\left(M_{Z}\right)$ & 0.1185 \\
\hline \hline
\end{tabular}

$\Gamma_{\text {tot }}^{\exp }=1.41_{-0.15}^{+0.19} \mathrm{GeV}, \quad F_{L}^{\exp }=0.687 \pm 0.018$,

$F_{-}^{\exp }=0.320 \pm 0.013$.

We study projections for higher integrated luminosities relevant for a high-luminosity LHC (HL-LHC) and a potential future $e^{+} e^{-}$collider later in this section. Since we perform a fit to only a limited set of observables, rather than a global fit such as considered in [27,29,30], our numerical results should only be considered representative of the achievable bounds on the studied operators.

\section{A. QCD operators}

We begin by discussing the contributions from QCD-like operators, namely $C_{t W}, C_{b W}, C_{\phi t b}, C_{t g}$, and $C_{b g}$. A similar analysis of these operators was performed in Ref. [31], ${ }^{2}$ focusing however only on the constraints derived for $C_{t W}$ and $C_{t g}$. A similar strategy has previously been employed for single top production and decay $[55,56]$. We update the constraints on these operators and discuss constraints on the remaining ones. At $\mathrm{LO}$, the total width is only a function of $C_{t W}, C_{b W}$, and $C_{\phi t b}$, which enter through the $\mathrm{W}$-vertex. The NLO corrections induce sensitivity to $C_{t g}$ comparable to that of $C_{\phi t b}$. We note that the total width is independent of $C_{b g}$, due to the operator being helicity suppressed, as evident from the analytic expression of Eq. (A2). We find that the total width is significantly more constraining for $C_{t W}$ than $C_{b W}$, and that the constraints on these two operators are both stronger than the bounds on $C_{\phi t b}$ and $C_{t g}$. The Wilson coefficients are also constrained through the longitudinal, positive transverse and negative transverse helicity fractions. The longitudinal rate $F_{L}$ is again independent of $C_{b g}$, as seen from Eq. (A3). We note that the variation of $C_{b W}$ significantly alters the positive transverse helicity fraction, $F_{+}$.

In order to derive constraints on the Wilson coefficients from the current experimental measurements of both the total width and the helicity fractions, we perform a oneparameter $\chi^{2}$ fit for each Wilson coefficient by keeping only one of them nonzero at a time. We also report projections for bounds potentially obtainable at a high luminosity LHC

\footnotetext{
${ }^{2}$ We find an identical analytic expressions for the total width $\Gamma_{\text {tot }}$ and longitudinal helicity fraction $F_{L}$ to leading order in $x_{b}$. We find however a different dependence on $C_{t g}$ in the case of the transverse helicity fractions $F_{ \pm}$.
}

TABLE II. Constraints on the Wilson coefficients of the QCD operators at $90 \%$ C.L. The scale $\Lambda$ is assumed to be $500 \mathrm{GeV}$. The first column shows the results based on the current LHC data with the luminosity of $20 \mathrm{fb}^{-1}$ and the rest of them the projection based on the HL-LHC with the luminosity of $3 \mathrm{ab}^{-1}$. For the projection of the uncertainties at HL-LHC, the statistical uncertainties scale like $\frac{1}{\sqrt{N}}$ while the systematic uncertainties are scaled by a factor of $f_{\text {syst }}$. For the projections, we assume that the experimental central values match the SM in order to focus on the achievable error reduction at the HL-LHC.

\begin{tabular}{lccc}
\hline \hline & Current & $\begin{array}{c}\text { HL-LHC } \\
\left(f_{\text {syst }}=1 / 2\right)\end{array}$ & $\begin{array}{c}\text { HL-LHC } \\
\left(f_{\text {syst }}=\frac{1}{\sqrt{N}}\right)\end{array}$ \\
\hline$C_{t W}$ & $-0.05<C_{t W}<0.17$ & $\left|C_{t W}\right|<0.04$ & $\left|C_{t W}\right|<0.01$ \\
$C_{t g}$ & $-13.37<C_{t g}<4.88$ & $\left|C_{t g}\right|<3.33$ & $\left|C_{t g}\right|<0.75$ \\
$C_{b W}$ & $-0.47<C_{b W}<2.07$ & $\left|C_{b W}\right|<0.45$ & $\left|C_{b W}\right|<0.10$ \\
$C_{b g}$ & $-32.72<C_{b g}<5.63$ & $\left|C_{b g}\right|<6.87$ & $\left|C_{b g}\right|<1.57$ \\
$C_{\phi t b}$ & $-4.15<C_{\phi t b}<12.84$ & $\left|C_{\phi t b}\right|<3.06$ & $\left|C_{\phi t b}\right|<0.69$ \\
\hline \hline
\end{tabular}

(HL-LHC) after collecting $3 \mathrm{ab}^{-1}$ of data. The $\chi^{2}$ function is defined through

$$
\chi^{2}=\sum_{i j}\left(O_{i}^{\text {theo }}-O_{i}^{\text {exp }}\right)\left(\sigma^{2}\right)_{i j}^{-1}\left(O_{j}^{\text {theo }}-O_{j}^{\exp }\right),
$$

where $O_{i}^{\exp }$ are the measured observables $\left(\Gamma_{\text {tot }}, F_{L}\right.$, and $\left.F_{-}\right), O_{i}^{\text {theo }}$ are their predicted values in the SMEFT and $\sigma_{i j}^{2}=\sigma_{i} \rho_{i j} \sigma_{j}$, where $\sigma_{i}$ are the uncertainties and $\rho$ is the correlation matrix,

$$
\rho=\left(\begin{array}{ccc}
1.0 & 0 & 0 \\
0 & 1.0 & -0.87 \\
0 & -0.87 & 1.0
\end{array}\right)
$$

The correlation matrix comes from a CMS measurement of the helicity fractions [57]. We assume that it is applicable to the PDG average and that the total width is uncorrelated with the $F_{i}$ measurements. We believe that these simple assumptions capture the features of a more complete analysis. For the asymmetric errors in $\Gamma_{\text {tot }}$, we combine them in quadrature; i.e., $\sigma_{\Gamma_{\text {tot }}}=\sqrt{\left(\sigma_{\Gamma_{\text {tot }}}^{\text {upper }}\right)^{2}+\left(\sigma_{\Gamma_{\text {tot }}}^{\text {lower }}\right)^{2}}$.

The results of the one-parameter fits are summarized in Table II. The first column of Table II shows the results based on the current LHC data with the luminosity of $20 \mathrm{fb}^{-1}$. The other columns show projections based on the HL-LHC with a luminosity of $3 \mathrm{ab}^{-1}$. For the projections we reduce the statistical errors as $1 / \sqrt{N}$, where the number of events $N$ scales like the integrated luminosity. We consider two assumptions for the scaling factor associated with the systematic error, $f_{\text {syst }}$.

(1) $f_{\text {syst }}=1 / 2$ : this is close to a recommendation proposed by ATLAS where all the systematic errors are scaled by a factor of $1 / 2[32,58]$. 

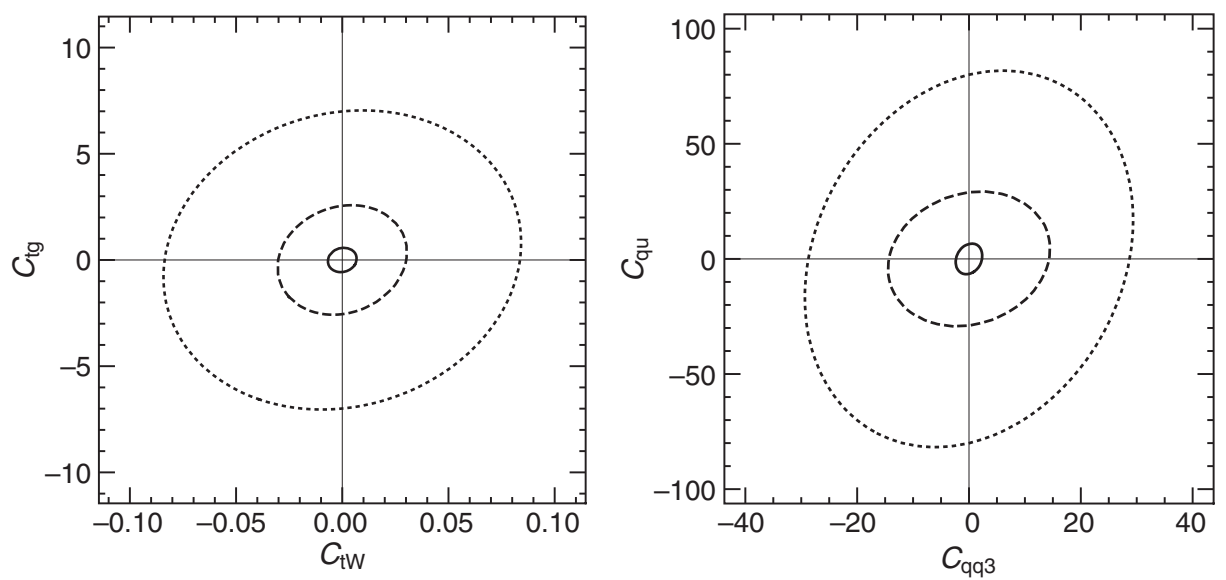

FIG. 4. Two-dimensional $\chi^{2}$ fit at $90 \%$ C.L. for the QCD operators, $C_{t W}$ and $C_{t g}$ (left), and the four-fermion operators, $C_{q q}^{(3)}$ and $C_{q u}$ (right). We shift the best-fit $C_{i}$ results to the origin in order to emphasize the improvement of the errors at the HL-LHC. We set $\Lambda=500 \mathrm{GeV}$ and show the dimensionless coefficient. The dotted contour corresponds to the current measurement, while the dashed and solid contours correspond to $f_{\text {syst }}=1 / 2$ and $f_{\text {syst }}=1 / \sqrt{N}$ at the HL-LHC.

(2) $f_{\text {syst }}=1 / \sqrt{N}$ : this is based on a CMS proposal used in previous projections [59], where the systematic error is assumed to scale like the statistical uncertainty. This is the more optimistic of the two scenarios.

We find that in the second projection the bounds on the QCD operators can be tightened by at least an order of magnitude at the HL-LHC, while in the first projection the uncertainty reduction is less. Both $C_{t W}$ and $C_{b W}$ are already significantly constrained with the current measurements. We have also performed a two-dimensional $\chi^{2}$ fit at 95\% C.L. for $C_{t W}$ and $C_{t g}$ as shown in the left panel of Fig. 4 to study potential correlations between these parameters. The dotted contour corresponds to the current measurement, while the dashed and solid contours correspond to $f_{\text {syst }}=1 / 2$ and $f_{\text {syst }}=1 / \sqrt{N}$ at the HL-LHC. Only a weak correlation is observed.

Previous constraints on these EFT operators at leading order using top quark observables can be found in the literature $[27,60]$. We have checked that when our calculation is truncated at LO the bounds we find agreement with those previously obtained. An important point learned from the above table is that at a HL-LHC, the bounds on the loop-induced Wilson coefficients $C_{t g}$ and $C_{b g}$ can approach unity. This demonstrates that higher-order effects in the SMEFT can be significantly probed during the future LHC program.

\section{B. Four-fermion operators}

We next present and discuss the bounds on the fourfermion operators to which we are sensitive: $C_{q u}^{(1)}, C_{q u}^{(8)}$, $C_{q q}^{(1)}, C_{q q}^{(3)}$, and $C_{l q}^{(3)}$. The sensitivity of the total width to the different Wilson coefficients is shown in Fig. 5. Since the observables we consider are only sensitive to the combination $C_{q u}=C_{q u}^{(1)}+\frac{4}{3} C_{q u}^{(8)}$ we plot only that structure. The shaded band represents the $1 \sigma$ region around the experimentally measured value, while the solid black line is the NLO result as a function of a single Wilson coefficient. We find with the current experimental errors that the total width is only weakly sensitive to these operators, with the exception of $C_{q q}^{(3)}$. We also find that $C_{l q}^{(3)}$ only appears in the total width and drops out from the helicity fractions after an expansion in $1 / \Lambda^{2} . C_{l q}^{(3)}$ is however only weakly bounded by the total width as is evident from Fig. 5 .

As before there are additional constraints set by the helicity fractions, $F_{L}$ and $F_{-}$. Figure 6 shows the helicity fractions as functions of $C_{q u}$. The shaded band is again the $1 \sigma$ region around the experimentally measured fractions. The solid black lines show the functional dependence of the helicity fractions on each Wilson coefficient at NLO. We see that the results are quite different than those observed for the total width. $C_{q u}$ is now probed by $F_{L}$, and $F_{-}$, but we lose sensitivity to all other four-fermion operators. We note that no observables are sensitive to $C_{q q}^{(1)}$ and $C_{l q}^{(3)}$.

The global constraints on the Wilson coefficients are derived through a one-parameter $\chi^{2}$ fit for each Wilson coefficient. The resulting best fits and corresponding bounds are summarized in Table III. We again compare the $68 \%$ C.L. bounds derived from current LHC data with the projected ones for HL-LHC at $3 \mathrm{ab}^{-1}$. As expected $C_{q q}^{(3)}$ is constrained most strongly. This bound is mainly set by the total width. The bounds on $C_{q u}$ are around a factor of 2 weaker in comparison, stemming from $F_{L}$ and $F_{-} . C_{q q}^{(1)}$ and $C_{l q}^{(3)}$ are not constrained through any of the observables. We have also performed a two-dimensional $\chi^{2}$ fit at 95\% C.L. for $C_{q q}^{(3)}$ and $C_{q u}$ as shown in the right panel of Fig 4. The dotted contour corresponds to the current 

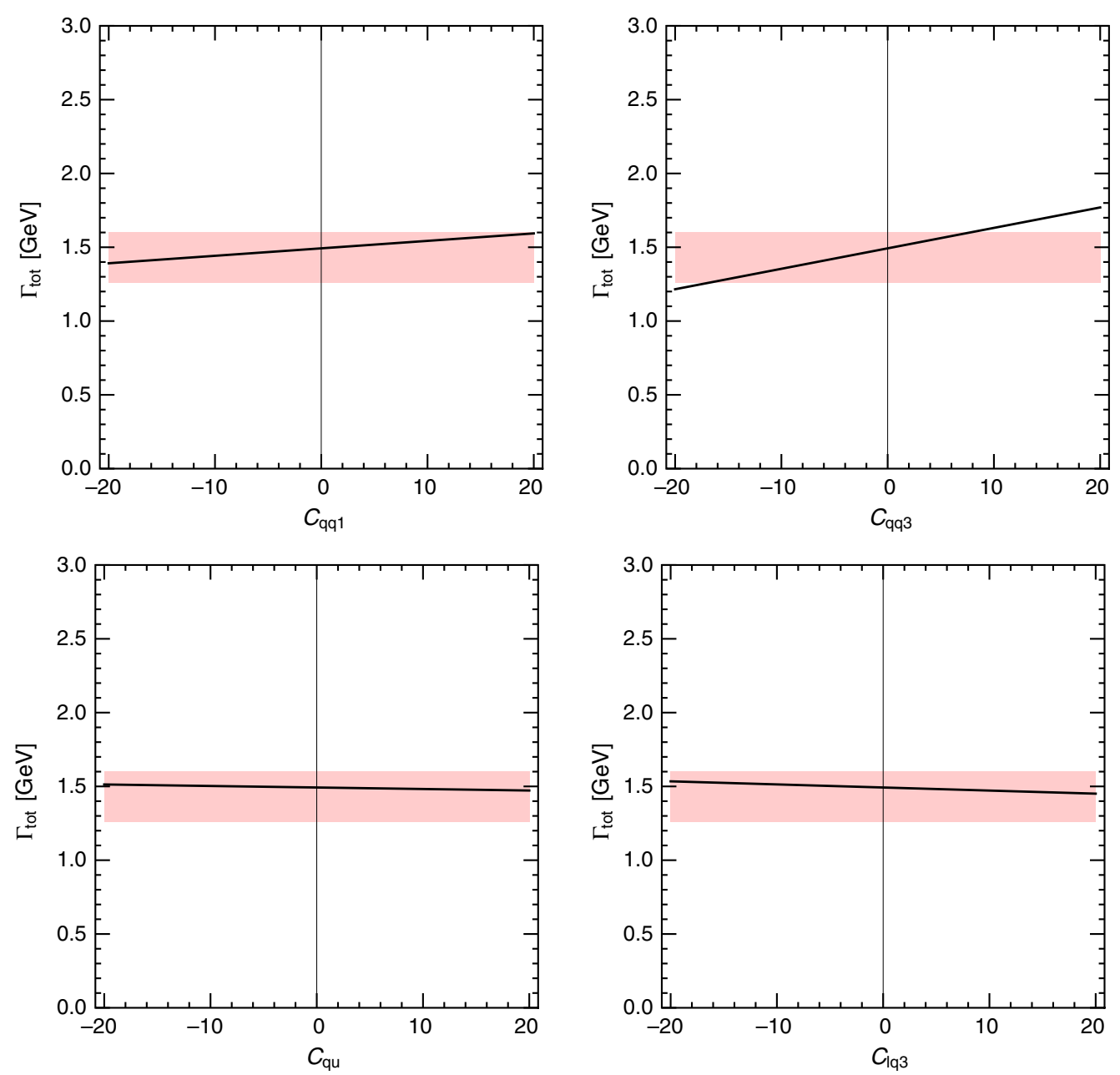

FIG. 5. Comparison of the Wilson coefficient sensitivity of the total decay width $\Gamma_{\text {tot }}$ for the four-fermion operators. We assume $\Lambda=500 \mathrm{GeV}$ and show the dimensionless coefficient. The shaded band shows the $1 \sigma$ region around the experimental decay width.

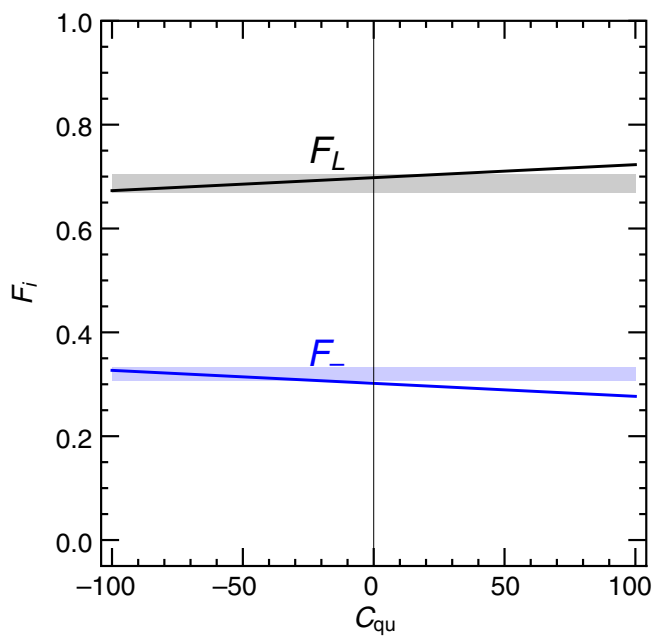

FIG. 6. Comparison of the Wilson coefficient sensitivity of the longitudinal and negative transversal helicity fractions for the four-fermion operators. We assume $\Lambda=500 \mathrm{GeV}$ and show the dimensionless coefficient. The shaded band shows the $1 \sigma$ region around the experimentally measured fractions. measurement, while the dashed and solid contours correspond to $f_{\text {syst }}=1 / 2$ and $f_{\text {syst }}=1 / \sqrt{N}$ at the HL-LHC. These bounds are complementary to the ones in the literature derived from direct production of four final-state heavy flavors [32].

We note that the bounds on all four-fermion Wilson coefficients are very weak with the current data. In fact, if we estimate the energy scale probed by each observable as $\Lambda / \sqrt{C_{X}}$, we find that the currently accessible energy scales are less than the top quark mass. This indicates that the EFT expansion is not compatible with the current experimental errors. At an HL-LHC, the bounds on all the four-fermion operators improve significantly. In particular, constraints on the Wilson coefficients $C_{q q}^{(3)}, C_{q u}^{(1)}$ and $C_{q u}^{(8)}$ approach unity and the effective energy scale probed is significantly above the top quark mass, indicating that these higher-order effects can be meaningfully probed during the future LHC program.

Finally, we study as well projected errors for a potential future FCC-ee $e^{+} e^{-}$machine. Details of this project are 
TABLE III. Constraints on the Wilson coefficients of the fourfermion operators with their respective errors at $90 \%$ C.L. The scale $\Lambda$ is assumed to be $500 \mathrm{GeV}$. The second column shows the results based on the current LHC data with the luminosity of $20 \mathrm{fb}^{-1}$ and the rest of them the projection based on the HL-LHC with the luminosity of $3 \mathrm{ab}^{-1}$. For the projection of the uncertainties at HL-LHC, the statistical uncertainties scale like $\frac{1}{\sqrt{N}}$ while the systematic uncertainties are scaled by a factor of $f_{\text {syst }}$. For the projections, we assume that the experimental central values match the SM in order to focus on the achievable error reduction at the HL-LHC.

\begin{tabular}{|c|c|c|c|}
\hline & Current & $\begin{array}{c}\text { HL-LHC } \\
\left(f_{\text {syst }}=1 / 2\right)\end{array}$ & $\begin{array}{c}\text { HL-LHC } \\
\left(f_{\text {syst }}=\frac{1}{\sqrt{N}}\right)\end{array}$ \\
\hline$C_{q q}^{(1)}$ & $-167.21<C_{q q}^{(1)}<254.73$ & $\left|C_{q q}^{(1)}\right|<105.48$ & $\left|C_{q q}^{(1)}\right|<17.23$ \\
\hline$C_{q q}^{(3)}$ & $-34.73<C_{q q}^{(3)}<22.80$ & $\left|C_{q q}^{(3)}\right|<14.38$ & $\left|C_{q q}^{(3)}\right|<2.35$ \\
\hline$C_{q u}$ & $-181.94<C_{q u}<26.91$ & $\left|C_{q u}\right|<37.80$ & $\left|C_{q u}\right|<8.53$ \\
\hline$C_{l q}^{(3)}$ & $-151.22<C_{l q}^{(3)}<230.36$ & $\left|C_{l q}^{(3)}\right|<95.40$ & $\left|C_{l q}^{(3)}\right|<15.58$ \\
\hline
\end{tabular}

provided in [61], where it is indicated that the top quark width can be probed with a precision of $45 \mathrm{MeV}$. We use this estimated error together with the more optimistic HL-LHC systematic error estimate to check what bounds the total width can provide on several example operators for each machine. The $68 \%$ C.L. uncertainties for the current measurement and the future colliders for $C_{q q}^{(3)}$ and $C_{t g}$ are shown in Fig. 7, where the blue, green, and red bars correspond to the uncertainties from the current measurement, FCC-ee, and HL-LHC $\left(f_{\text {syst }}=1 / \sqrt{N}\right)$, respectively. We find that the bounds are significantly improved at both future colliders.

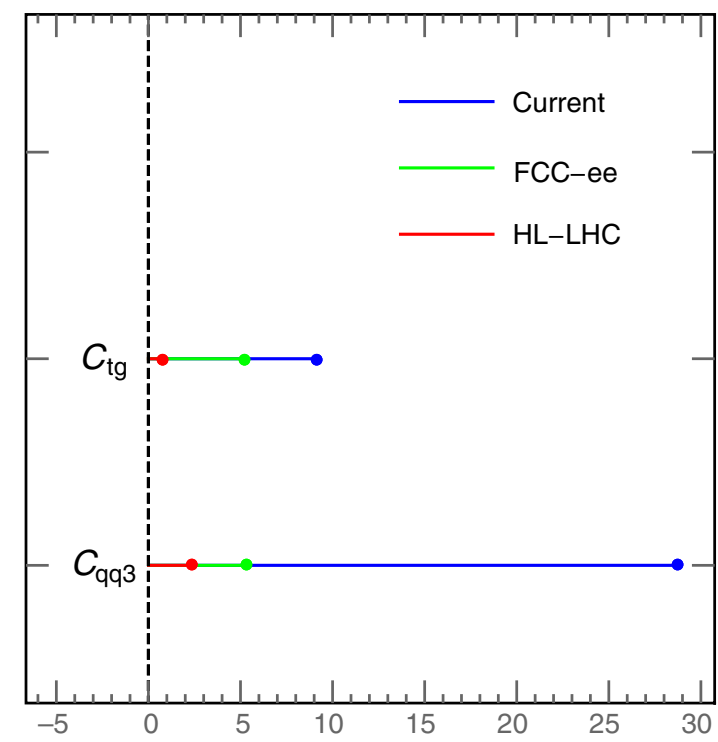

FIG. 7. $90 \%$ C.L. uncertainties for the QCD operator, $C_{t g}$, and four-fermion operator, $C_{q q}^{(3)}$. The blue, green, and red bars correspond to the uncertainties from the current measurement, FCC-ee, and HL-LHC $\left(f_{\text {syst }}=1 / \sqrt{N}\right)$, respectively.

\section{CONCLUSIONS}

In this manuscript we have studied the next-to-leading order corrections to top quark decays within the SMEFT. Our calculation includes a more complete set of operators than previously studied, in particularly all contributing four-fermion operators. We have two primary motivations. First, this work is a step toward a complete calculation of NLO effects within SMEFT, which we believe will eventually be required by the experimental uncertainties. Second, this work tests the question of whether loopinduced operators can be probed with either current or potential future collider data.

We have addressed technical aspects associated with higher-order calculations in the SMEFT containing $\gamma_{5}$. Chiral Ward identities play an important role in imposing consistency of $\gamma_{5}$ prescriptions at higher order in dimensional regularization, which we demonstrate by checking the consistency of several different schemes at one-loop order. Finally, we have presented numerical bounds on the considered operators given current and projected future uncertainties. We find that future machines such as the HL-LHC or a future $e^{+} e^{-}$collider can provide important constraints on SMEFT operators that first appear at higher orders.

\section{ACKNOWLEDGMENTS}

We thank C. Zhang for useful discussions. R. B. is supported by the DOE Contract No. DE-AC0206CH11357. C.-Y.C. is supported by the NSF Grant No. NSF-1740142. F. P. and D. W. are supported by the DOE Grants No. DE-FG02-91ER40684 and No. DEAC02-06CH11357. This research used resources of the Argonne Leadership Computing Facility, which is a DOE Office of Science User Facility supported under Contract No. DE-AC02-06CH11357.

\section{APPENDIX: NLO EXPRESSIONS FOR THE HELICITY FRACTIONS}

We present here the analytic results for the NLO SMEFT corrected helicity fractions $F_{i}$ and the total decay width $\Gamma_{\text {tot }}$ of $t \rightarrow W b$. For convenience we split up the different contributions.

$$
\begin{aligned}
\Gamma_{\mathrm{tot}} & =\Gamma_{\mathrm{Born}}+\Delta \Gamma_{\mathrm{QCD}}+\Delta \Gamma_{4 \mathrm{f}}, \\
F_{i} & =F_{i}^{\mathrm{Born}}+\Delta F_{i}^{\mathrm{QCD}}+\Delta F_{i}^{4 \mathrm{f}},
\end{aligned}
$$

where the first term in the expansion describes the contributions coming from the tree-level diagrams only (with full $x_{b}$ dependence retained), while the second one contains all QCD-like corrections expanded up to order $g_{s}^{2}$ and the last the pieces from the four-Fermi operators. We consistently expanded all three contributions to leading order in $\frac{1}{\Lambda^{2}}$. 


\section{QCD corrected decay fractions}

$$
\begin{aligned}
\Delta \Gamma_{\mathrm{QCD}}= & -\frac{g_{s} \bar{g} m_{t}}{2304 \pi^{3} x_{W}^{2}}\left[( x _ { W } ^ { 2 } - 1 ) \left(6 \sqrt{2} C_{t g} \bar{g} m_{t}^{2} x_{v}\left(1+11 x_{W}^{2}-20 x_{W}^{4}\right)\right.\right. \\
& +g_{s}\left(12 \sqrt{2} C_{t W} m_{t}^{2} x_{v} x_{W}^{2}\left(17-21 x_{W}^{2}+4 \pi^{2}\left(x_{W}^{2}-1\right)\right)+\bar{g}\left(3\left(5+9 x_{W}^{2}-6 x_{W}^{4}\right)\right.\right. \\
& \left.\left.\left.+\pi^{2}\left(8 x_{W}^{4}-4-4 x_{W}^{2}\right)\right)\right)\right)+\log \left(1-x_{W}\right)\left(6 ( - 1 + x _ { W } ^ { 2 } ) ^ { 2 } \left(4 \sqrt{2} C_{t g} \bar{g} m_{t}^{2} x_{v}\left(x_{W}^{2}-1\right)\right.\right. \\
& \left.+g_{s}\left(\bar{g}\left(5+4 x_{W}^{2}\right)+4 \sqrt{2} C_{t W} m_{t}^{2} x_{v}\left(2+7 x_{W}^{2}\right)\right)\right)+24 g_{s}\left(x_{W}^{2}-1\right)^{2}\left(\bar{g}\left(1+2 x_{W}^{2}\right)\right. \\
& \left.\left.+12 \sqrt{2} C_{t W} m_{t}^{2} x_{v} x_{W}^{2}\right) \log \left(x_{W}\right)\right)+6\left(x_{W}^{2}-1\right)^{2}\left(4 \sqrt{2} C_{t g} \bar{g} m_{t}^{2} x_{v}\left(x_{W}^{2}-1\right)\right. \\
& \left.+g_{s}\left(\bar{g}\left(5+4 x_{W}^{2}\right)+4 \sqrt{2} C_{t W} m_{t}^{2} x_{v}\left(2+7 x_{W}^{2}\right)\right)\right) \log \left(1+x_{W}\right) \\
& +\left(2 4 x _ { W } ^ { 2 } \left(\sqrt{2} C_{t g} \bar{g} m_{t}^{2} x_{v} x_{W}^{2}\left(3+x_{W}^{2}\right)+g_{s}\left(4 \sqrt{2} C_{t W} m_{t}^{2} x_{v} x_{W}^{2}\left(3-2 x_{W}^{2}\right)\right.\right.\right. \\
& \left.\left.\left.-\bar{g}\left(x_{W}^{2}+2 x_{W}^{4}-1\right)\right)\right)\right) \log \left(x_{W}\right)+48 g_{s}\left(x_{W}^{2}-1\right)^{2}\left(\bar{g}\left(1+2 x_{W}^{2}\right)\right. \\
& \left.\left.+12 \sqrt{2} C_{t W} m_{t}^{2} x_{v} x_{W}^{2}\right)\left(\operatorname{Li}_{2}\left(x_{W}\right)+\operatorname{Li}_{2}\left(-x_{W}\right)+\frac{1}{2} \log \left(x_{W}\right) \log \left(1+x_{W}\right)\right)\right]
\end{aligned}
$$

$$
\begin{aligned}
\Delta F_{\mathrm{L}}^{\mathrm{QCD}}= & \frac{g_{s}}{9 \bar{g} \pi^{2}\left(1-x_{W}^{2}\right)^{2}\left(1+2 x_{W}^{2}\right)^{3}}\left[x _ { W } ^ { 2 } \left(-\sqrt{2} C_{t g} \bar{g} m_{t}^{2} x_{v}\left(1+2 x_{W}^{2}\right)\left(-6 x_{W}^{2}\left(9-10 x_{W}^{2}+x_{W}^{4}\right)\right.\right.\right. \\
& \left.+\pi^{2}\left(1+5 x_{W}^{2}+6 x_{W}^{4}\right)\right)+g_{s}\left(\overline { g } ( 1 + 2 x _ { W } ^ { 2 } ) \left(-\pi^{2}\left(7+15 x_{W}^{2}+2 x_{W}^{4}\right)+6\left(6+6 x_{W}^{2}\right.\right.\right. \\
& \left.\left.-13 x_{W}^{4}+x_{W}^{6}\right)\right)+2 \sqrt{2} C_{t W} m_{t}^{2} x_{v}\left(\pi^{2}\left(1+49 x_{W}^{2}+106 x_{W}^{4}+24 x_{W}^{6}\right)+6\left(-2-39 x_{W}^{2}\right.\right. \\
& \left.\left.\left.\left.-40 x_{W}^{4}+79 x_{W}^{6}+2 x_{W}^{8}\right)\right)\right)\right)+\left(3 ( 1 - x _ { W } ^ { 2 } ) ^ { 3 } \left(\sqrt{2} C_{t g} \bar{g} m_{t}^{2} x_{v}\left(4 x_{W}^{4}-1\right)\right.\right. \\
& \left.+g_{s}\left(\bar{g}+2 \bar{g} x_{W}^{2}+2 \sqrt{2} C_{t W} m_{t}^{2} x_{v}\left(1-10 x_{W}^{2}\right)\right)\right)+3\left(1-x_{W}\right)^{3} x_{W}\left(1+2 x_{W}^{2}\right)\left(g _ { s } \left(4 \sqrt { 2 } \left(1-15 x_{W}\right.\right.\right. \\
& \left.\left.\left.\left.-x_{W}^{2}\right) C_{t W} m_{t}^{2} x_{v} x_{W}+\bar{g}\left(5+x_{W}+10 x_{W}^{2}+2 x_{W}^{3}\right)\right)-2 \sqrt{2} C_{t g} \bar{g} m_{t}^{2} x_{v} x_{W}\left(1+2 x_{W}^{2}\right)\right) \log \left(x_{W}\right)\right) \log \left(1-x_{W}\right) \\
& -3\left(-1+x_{W}^{2}\right)^{3}\left(\sqrt{2} C_{t g} \bar{g} m_{t}^{2} x_{v}\left(4 x_{W}^{4}-1\right)+g_{s}\left(\bar{g}+2 \bar{g} x_{W}^{2}+2 \sqrt{2} C_{t W} m_{t}^{2} x_{v}\left(1-10 x_{W}^{2}\right)\right)\right) \log \left(1+x_{W}\right) \\
& +\left(6 x _ { W } ^ { 2 } \left(\sqrt{2} C_{t g} \bar{g} m_{t}^{2} x_{v} x_{W}^{2}\left(7+23 x_{W}^{2}+18 x_{W}^{4}\right)-g_{s}\left(2 \sqrt { 2 } C _ { t W } m _ { t } ^ { 2 } x _ { v } x _ { W } ^ { 2 } \left(35+101 x_{W}^{2}\right.\right.\right.\right. \\
& \left.\left.\left.+112 x_{W}^{4}+4 x_{W}^{6}\right)-\bar{g}\left(5+25 x_{W}^{2}+44 x_{W}^{4}+28 x_{W}^{6}\right)\right)\right) \\
& -3 x_{W}\left(1+x_{W}\right)^{3}\left(1+2 x_{W}^{2}\right)\left(2 \sqrt{2} C_{t g} \bar{g} m_{t}^{2} x_{v} x_{W}\left(1+2 x_{W}^{2}\right)-g_{s}\left(4 \sqrt{2} C_{t W} m_{t}^{2} x_{v} x_{W}(1\right.\right. \\
& \left.\left.\left.\left.+15 x_{W}-x_{W}^{2}\right)+\bar{g}\left(5-x_{W}+10 x_{W}^{2}-2 x_{W}^{3}\right)\right)\right) \log \left(1+x_{W}\right)\right) \log \left(x_{W}\right) \\
& +3 x_{W}\left(1+x_{W}\right)^{3}\left(1+2 x_{W}^{2}\right)\left(-2 \sqrt{2} C_{t g} \bar{g} m_{t}^{2} x_{v} x_{W}\left(1+2 x_{W}^{2}\right)\right. \\
& +g_{s}\left(-4 \sqrt{2} C_{t W} m_{t}^{2} x_{v} x_{W}\left(-1-15 x_{W}+x_{W}^{2}\right)+\bar{g}\left(-5+x_{W}-10 x_{W}^{2}\right.\right. \\
& \left.\left.\left.+2 x_{W}^{3}\right)\right)\right) \operatorname{Li}_{2}\left(-x_{W}\right)+3\left(1-x_{W}\right)^{3} x_{W}\left(1+2 x_{W}^{2}\right)\left(-2 \sqrt{2} C_{t g} \bar{g} m_{t}^{2} x_{v} x_{W}\left(1+2 x_{W}^{2}\right)\right. \\
& \left.\left.+g_{s}\left(-4 \sqrt{2} C_{t W} m_{t}^{2} x_{v} x_{W}\left(-1+15 x_{W}+x_{W}^{2}\right)+\bar{g}\left(5+x_{W}+10 x_{W}^{2}+2 x_{W}^{3}\right)\right)\right) \mathrm{Li}{ }_{2}\left(x_{W}\right)\right]
\end{aligned}
$$

$$
\begin{aligned}
\Delta F_{-}^{\mathrm{QCD}}= & \frac{g_{s}}{36 \bar{g} \pi^{2}\left(1-x_{W}^{2}\right)^{2}\left(1+2 x_{W}^{2}\right)^{3}}\left[x _ { W } ^ { 2 } \left(6 \sqrt { 2 } \overline { g } m _ { t } ^ { 2 } x _ { v } ( 1 - x _ { W } + 2 x _ { W } ^ { 2 } - 2 x _ { W } ^ { 3 } ) \left(( 1 + x _ { W } ) ^ { 2 } \left(x_{W}\right.\right.\right.\right. \\
& \left.\left.-2 x_{W}^{2}+2 x_{W}^{3}-1\right) C_{b g}+2 C_{t g}\left(2-3 x_{W}-6 x_{W}^{2}-23 x_{W}^{3}+18 x_{W}^{4}+4 x_{W}^{5}\right)\right) \\
& +g_{s}\left(\overline { g } ( 1 + 2 x _ { W } ^ { 2 } ) \left(2 \pi^{2}\left(7+10 x_{W}^{2}-6 x_{W}^{4}+4 x_{W}^{6}\right)-3\left(23+20 x_{W}-6 x_{W}^{2}+48 x_{W}^{3}\right.\right.\right. \\
& \left.\left.-111 x_{W}^{4}+16 x_{W}^{5}+10 x_{W}^{6}\right)\right)-4 \sqrt{2} C_{t W} m_{t}^{2} x_{v}\left(3 \left(-8+30 x_{W}-133 x_{W}^{2}+82 x_{W}^{3}\right.\right. \\
& \left.-206 x_{W}^{4}+64 x_{W}^{5}+129 x_{W}^{6}+40 x_{W}^{7}+2 x_{W}^{8}\right)+2 \pi^{2}\left(1+33 x_{W}^{2}+64 x_{W}^{4}+6 x_{W}^{6}\right. \\
& \left.\left.\left.\left.+4 x_{W}^{8}\right)\right)\right)\right)+\left(1 2 ( x _ { W } ^ { 2 } - 1 ) ^ { 3 } \left(\sqrt{2} C_{t g} \bar{g} m_{t}^{2} x_{v}\left(4 x_{W}^{4}-1\right)+g_{s}\left(\bar{g}+2 \bar{g} x_{W}^{2}\right.\right.\right.
\end{aligned}
$$




$$
\begin{aligned}
& \left.\left.+2 \sqrt{2} C_{t W} m_{t}^{2} x_{v}\left(1-10 x_{W}^{2}\right)\right)\right)+6\left(-1+x_{W}\right)^{2} x_{W}\left(1+2 x_{W}^{2}\right)\left(2 \sqrt{2} C_{t g} \bar{g} m_{t}^{2} x_{v} x_{W}(1\right. \\
& \left.-x_{W}+2 x_{W}^{2}-2 x_{W}^{3}\right)+g_{s}\left(-4 \sqrt{2} C_{t W} m_{t}^{2} x_{v} x_{W}\left(-3-24 x_{W}+14 x_{W}^{2}+9 x_{W}^{3}+4 x_{W}^{4}\right)\right. \\
& \left.\left.\left.-\bar{g}\left(5-8 x_{W}+x_{W}^{2}-20 x_{W}^{3}-18 x_{W}^{4}-8 x_{W}^{5}\right)\right)\right) \log \left(x_{W}\right)\right) \log \left(1-x_{W}\right) \\
& -12\left(1-x_{W}^{2}\right)\left(-\sqrt{2} C_{t g} \bar{g} m_{t}^{2} x_{v}\left(1+x_{W}^{2}+10 x_{W}^{4}+24 x_{W}^{6}\right)+g_{s}\left(\overline { g } \left(1-5 x_{W}^{2}-21 x_{W}^{4}\right.\right.\right. \\
& \left.\left.\left.-10 x_{W}^{6}+8 x_{W}^{8}\right)+2 \sqrt{2} C_{t W} m_{t}^{2} x_{v}\left(1-25 x_{W}^{2}+2 x_{W}^{4}-2 x_{W}^{6}-12 x_{W}^{8}\right)\right)\right) \log \left(1+x_{W}\right) \\
& -\left(1 2 x _ { W } ^ { 2 } \left(\sqrt{2} C_{t g} \bar{g} m_{t}^{2} x_{v} x_{W}^{2}\left(7+17 x_{W}^{2}+4 x_{W}^{4}-4 x_{W}^{6}\right)+g_{s}\left(-2 \sqrt{2} C_{t W} m_{t}^{2} x_{v} x_{W}^{2}(39\right.\right.\right. \\
& \left.\left.\left.+93 x_{W}^{2}+136 x_{W}^{4}+20 x_{W}^{6}\right)+\bar{g}\left(5+23 x_{W}^{2}+42 x_{W}^{4}+36 x_{W}^{6}+8 x_{W}^{8}\right)\right)\right) \\
& -6 x_{W}\left(1+x_{W}\right)^{2}\left(1+2 x_{W}^{2}\right)\left(2 \sqrt{2} C_{t g} \bar{g} m_{t}^{2} x_{v} x_{W}\left(1+x_{W}+2 x_{W}^{2}+2 x_{W}^{3}\right)\right. \\
& +g_{s}\left(4 \sqrt{2} C_{t W} m_{t}^{2} x_{v} x_{W}\left(3-24 x_{W}-14 x_{W}^{2}+9 x_{W}^{3}-4 x_{W}^{4}\right)+\bar{g}\left(5+8 x_{W}+x_{W}^{2}\right.\right. \\
& \left.\left.\left.\left.+20 x_{W}^{3}-18 x_{W}^{4}+8 x_{W}^{5}\right)\right)\right) \log \left(1+x_{W}\right)\right) \log \left(x_{W}\right) \\
& +6 x_{W}\left(1+2 x_{W}^{2}\right)\left(2 \sqrt{2} C_{t g} \bar{g} m_{t}^{2} x_{v}\left(x_{W}-1\right)^{3} x_{W}\left(1+2 x_{W}^{2}\right)+g_{s}\left(4 \sqrt{2} C_{t W} m_{t}^{2} x_{v} x_{W}(1\right.\right. \\
& \left.-18 x_{W}-89 x_{W}^{2}-43 x_{W}^{3}+16 x_{W}^{4}+x_{W}^{5}-12 x_{W}^{6}\right)+\bar{g}\left(5+18 x_{W}+22 x_{W}^{2}+10 x_{W}^{3}\right. \\
& \left.\left.\left.+23 x_{W}^{4}-40 x_{W}^{5}-2 x_{W}^{6}+24 x_{W}^{7}\right)\right)\right) \operatorname{Li}_{2}\left(-x_{W}\right) \\
& +6\left(1-x_{W}\right)^{2} x_{W}\left(1+2 x_{W}^{2}\right)\left(2 \sqrt{2} C_{t g} \bar{g} m_{t}^{2} x_{v} x_{W}\left(1-x_{W}+2 x_{W}^{2}-2 x_{W}^{3}\right)\right. \\
& +g_{s}\left(4 \sqrt{2} C_{t W} m_{t}^{2} x_{v} x_{W}\left(3+24 x_{W}-14 x_{W}^{2}-9 x_{W}^{3}-4 x_{W}^{4}\right)-\bar{g}\left(5-8 x_{W}\right.\right. \\
& \left.\left.\left.\left.+x_{W}^{2}-20 x_{W}^{3}-18 x_{W}^{4}-8 x_{W}^{5}\right)\right)\right) \operatorname{Li}_{2}\left(x_{W}\right)\right] \\
& +
\end{aligned}
$$

\section{Four-fermion corrected decay fractions}

Here we report the four-Fermi corrected longitudinal and negative transverse helicity fractions, and the total decay width of $t \rightarrow W b$. We omit the contributions stemming from the $t-b-u-d$ vertex leading to two massless quarks in the loop.

$$
\begin{aligned}
\Delta \Gamma_{4 \mathrm{f}}= & \frac{\bar{g} m_{T}^{3}\left(x_{W}^{2}-1\right)^{2}}{4608 \pi^{2} x_{W}^{6}}\left[12 C_{l q}^{(3)} x_{W}^{6}\left(1+2 x_{W}^{2}\right) \log \left(x_{W}^{2}\right)-x_{W}^{2}\left(x _ { W } ^ { 4 } \left(20 C_{l q}^{(3)}+27 C_{q u}^{(1)}+36 C_{q u}^{(8)}\right.\right.\right. \\
& \left.+40 C_{l q}^{(3)} x_{W}^{2}\right)+2 C_{q q}^{(1)}\left(-3-3 x_{W}^{2}+10 x_{W}^{4}+8 x_{W}^{6}\right)+2 C_{q q}^{(3)}\left(-15-69 x_{W}^{2}-22 x_{W}^{4}\right. \\
& \left.\left.\left.+112 x_{W}^{6}\right)\right)+6\left(C_{q q}^{(1)}+5 C_{q q}^{(3)}\right)\left(1+x_{W}^{2}-2 x_{W}^{4}\right)^{2} \log \left(1-x_{W}^{2}\right)\right] \\
\Delta F_{\mathrm{L}}^{4 \mathrm{f}}= & \frac{m_{t}^{2} x_{W}^{2}}{12 \pi^{2}} \frac{\left(x_{W}^{2}-1\right)}{\left(1+2 x_{W}^{2}\right)^{2}}\left(3 C_{q u}^{(1)}+4 C_{q u}^{(8)}\right) \\
\Delta F_{-}^{4 \mathrm{f}}= & -\frac{m_{t}^{2} x_{W}^{2}}{12 \pi^{2}} \frac{\left(x_{W}^{2}-1\right)}{\left(1+2 x_{W}^{2}\right)^{2}}\left(3 C_{q u}^{(1)}+4 C_{q u}^{(8)}\right) .
\end{aligned}
$$

[1] W. Buchmuller and D. Wyler, Nucl. Phys. B268, 621 (1986).

[2] B. Grzadkowski, M. Iskrzynski, M. Misiak, and J. Rosiek, J. High Energy Phys. 10 (2010) 085.

[3] Z. Han and W. Skiba, Phys. Rev. D 71, 075009 (2005).
[4] A. Pomarol and F. Riva, J. High Energy Phys. 01 (2014) 151.

[5] C. Y. Chen, S. Dawson, and C. Zhang, Phys. Rev. D 89, 015016 (2014).

[6] J. Ellis, V. Sanz, and T. You, J. High Energy Phys. 07 (2014) 036. 
[7] J. D. Wells and Z. Zhang, Phys. Rev. D 90, 033006 (2014).

[8] A. Falkowski and F. Riva, J. High Energy Phys. 02 (2015) 039.

[9] J. de Blas, M. Ciuchini, E. Franco, S. Mishima, M. Pierini, L. Reina, and L. Silvestrini, J. High Energy Phys. 12 (2016) 135.

[10] C. Hartmann and M. Trott, J. High Energy Phys. 07 (2015) 151.

[11] C. Hartmann and M. Trott, Phys. Rev. Lett. 115, 191801 (2015).

[12] A. Dedes, M. Paraskevas, J. Rosiek, K. Suxho, and L. Trifyllis, J. High Energy Phys. 08 (2018) 103.

[13] R. Gauld, B. D. Pecjak, and D. J. Scott, J. High Energy Phys. 05 (2016) 080.

[14] R. Gauld, B. D. Pecjak, and D. J. Scott, Phys. Rev. D 94, 074045 (2016).

[15] J. M. Cullen, B. D. Pecjak, and D. J. Scott, J. High Energy Phys. 08 (2019) 173.

[16] S. Dawson and P. P. Giardino, Phys. Rev. D 97, 093003 (2018).

[17] S. Dawson and P. P. Giardino, Phys. Rev. D 98, 095005 (2018).

[18] C. Hartmann, W. Shepherd, and M. Trott, J. High Energy Phys. 03 (2017) 060.

[19] S. Dawson and A. Ismail, Phys. Rev. D 98, 093003 (2018).

[20] S. Dawson, P. P. Giardino, and A. Ismail, Phys. Rev. D 99, 035044 (2019).

[21] C. Degrande, J. M. Gerard, C. Grojean, F. Maltoni, and G. Servant, J. High Energy Phys. 07 (2012) 036; 03 (2013) 032(E).

[22] E. Vryonidou and C. Zhang, J. High Energy Phys. 08 (2018) 036.

[23] L. Berthier and M. Trott, J. High Energy Phys. 05 (2015) 024.

[24] G. Passarino and M. Trott, arXiv:1610.08356.

[25] N. Greiner, S. Willenbrock, and C. Zhang, Phys. Lett. B 704, 218 (2011).

[26] C. Zhang, N. Greiner, and S. Willenbrock, Phys. Rev. D 86, 014024 (2012).

[27] A. Buckley, C. Englert, J. Ferrando, D. J. Miller, L. Moore, M. Russell, and C. D. White, J. High Energy Phys. 04 (2016) 015.

[28] V. Cirigliano, W. Dekens, J. de Vries, and E. Mereghetti, Phys. Rev. D 94, 034031 (2016).

[29] J. A. Aguilar-Saavedra et al., arXiv:1802.07237.

[30] N. P. Hartland, F. Maltoni, E. R. Nocera, J. Rojo, E. Slade, E. Vryonidou, and C. Zhang, J. High Energy Phys. 04 (2019) 100.

[31] C. Zhang, Phys. Rev. D 90, 014008 (2014).

[32] P. Azzi et al. (HL-LHC Collaboration and HE-LHC Working Group), arXiv:1902.04070.
[33] A. Dedes, W. Materkowska, M. Paraskevas, J. Rosiek, and K. Suxho, J. High Energy Phys. 06 (2017) 143.

[34] E. E. Jenkins, A. V. Manohar, and M. Trott, J. High Energy Phys. 10 (2013) 087.

[35] E. E. Jenkins, A. V. Manohar, and M. Trott, J. High Energy Phys. 01 (2014) 035.

[36] R. Alonso, E. E. Jenkins, A. V. Manohar, and M. Trott, J. High Energy Phys. 04 (2014) 159.

[37] M. Fischer, S. Groote, J. G. Korner, and M. C. Mauser, Phys. Rev. D 63, 031501 (2001).

[38] H. S. Do, S. Groote, J. G. Korner, and M. C. Mauser, Phys. Rev. D 67, 091501 (2003).

[39] K. G. Chetyrkin and F. V. Tkachov, Nucl. Phys. B192, 159 (1981).

[40] F. Jegerlehner, Eur. Phys. J. C 18, 673 (2001).

[41] T. L. Trueman, Z. Phys. C 69, 525 (1996).

[42] S. A. Larin, Phys. Lett. B 303, 113 (1993).

[43] P. Breitenlohner and D. Maison, Commun. Math. Phys. 52, 39 (1977).

[44] J. G. Korner, D. Kreimer, and K. Schilcher, Z. Phys. C 54, 503 (1992).

[45] D. Kreimer, arXiv:hep-ph/9401354.

[46] D. J. Broadhurst and A. G. Grozin, Phys. Rev. D 52, 4082 (1995).

[47] J. F. Donoghue, E. Golowich, and B. R. Holstein, Cambridge Monogr. Part. Phys., Nucl. Phys., Cosmol. 2, 1 (1992); 35, 82 (2014), Section III-3.

[48] V. Shtabovenko, J. Phys. Conf. Ser. 762, 012064 (2016).

[49] A. V. Bednyakov, A. F. Pikelner, and V. N. Velizhanin, J. Phys. Conf. Ser. 523, 012045 (2014); Phys. Lett. B 722, 336 (2013); D. Stöckinger, Dimensional regularization and $\gamma_{5}$, Proceedings of the FCCee (2018) [arXiv:1809 .01830].

[50] M. Jamin and M.E. Lautenbacher, Comput. Phys. Commun. 74, 265 (1993).

[51] K. Adel and Y. P. Yao, Phys. Rev. D 53, 374 (1996).

[52] S. Herrlich and U. Nierste, Nucl. Phys. B455, 39 (1995).

[53] W. E. Caswell, Phys. Rev. Lett. 33, 244 (1974).

[54] M. Tanabashi et al. (Particle Data Group), Phys. Rev. D 98, 030001 (2018).

[55] M. de Beurs, E. Laenen, M. Vreeswijk, and E. Vryonidou, Eur. Phys. J. C 78, 919 (2018).

[56] T. Neumann and Z. E. Sullivan, J. High Energy Phys. 06 (2019) 022.

[57] V. Khachatryan et al. (CMS Collaboration), Phys. Lett. B 762, 512 (2016).

[58] ATLAS Collaboration, Report No. ATL-PHYS-PUB-2019005.

[59] CMS Collaboration, Report No. CMS-NOTE-2018-006.

[60] C. Zhang and S. Willenbrock, Phys. Rev. D 83, 034006 (2011).

[61] A. Abada et al. (FCC Collaboration), Report No. CERNACC-2018-0057. 